\title{
Liberale Demokratien und der Zwang zum Dienst an der Waffe
}

\author{
Sebastian SCHIEF ${ }^{1}$ \\ Universität Fribourg
}

\begin{abstract}
Einleitung
Es gibt eine interessante Konstellation innerhalb liberaler westlicher Gesellschaften, die häufig zu Kontroversen geführt hat, weil sich zwei Interpretationen von Liberalismus diametral gegenüberstehen. Die Rede ist von Zwangsdienst von (zumeist) Männern im Militär einer liberalen Demokratie. Die Konfliktlinie verläuft hier zwischen einerseits der Interpretation, dass der Staat in liberalen Gesellschaften den Bürger und die Bürgerin nicht zu Zwangsdiensten jeglicher Art zwingen könne, weil dies ein unbilliger Eingriff in die Freiheit des Citoyens sei. Andererseits verteidigen die Befürworterinnen und Befürworter des Zwangsdienstes diesen mit Verweis auf die Notwendigkeit der Verteidigung liberaler Gesellschaften. Für die Verteidigung der freien Gesellschaft sei es schlicht notwendig, dass Personen zum Dienst an der Waffe gezwungen würden. Es gäbe dementsprechend einen individuellen Zwang, der sich aus dem zu verteidigenden Ideal einer freien Gesellschaft ableiten lasse. Im Folgenden werde ich zuerst drei Beispiele für Zwangsdienste an der Waffe vorstellen und diskutieren: die Wehrpflicht in Deutschland nach der Einführung der Bundeswehr, die Wehrpflicht in den USA und die Wehrpflicht in der Schweiz. Im Anschluss diskutiere ich die Durchsetzung des Zwangs in der Schweiz detaillierter. Ich werde zeigen, dass mit dem Ziel der Verteidigung liberaler Gesellschaften die Citoyens innerhalb dieser mitunter ihrer Bürgerrechte beraubt wurden und Diskriminierungen ausgesetzt waren. Dies, ohne dass je eine Entschuldigung oder Wiedergutmachung durch den Staat erfolgte.
\end{abstract}

\section{Wehrpflicht in Deutschland}

Nach dem Zweiten Weltkrieg hatte die Bundesrepublik Deutschland aus nachvollziehbaren Gründen zunächst keine Armee. Die Frage der Wiederbewaffnung war hochgradig umstritten. Dies kann auch an der Entstehung einer Bewegung gegen die Wiederbewaffnung gesehen werden (Narr 2008). Erst nach dem Abschluss der Pariser Verträge 1955 wurde die Bundeswehr aufgebaut. Diese war zunächst noch eine Freiwilligenarmee. Die allgemeine Wehrpflicht wurde im Juli 1956 verankert, erste Rekrutierungen begannen im Januar 1957. Von Anfang an war im Wehrpflichtgesetz (WPflG) vom 21. Juli 1956 allerdings das Recht auf Verweigerung des Wehrdienstes verankert:

„Wer sich aus Gewissensgründen der Beteiligung an jeder Waffenanwendung zwischen den Staaten widersetzt und deshalb den Kriegsdienst mit der Waffe verweigert, hat statt des

\footnotetext{
${ }^{1}$ Dr. Sebastian Schief ist Lehr- und Forschungsrat am Departement Sozialwissenschaften der Universität Freiburg.
} 
Wehrdienstes einen zivilen Ersatzdienst ausserhalb der Bundeswehr zu leisten. Er kann auf seinen Antrag zum waffenlosen Dienst in der Bundeswehr herangezogen werden. " $(\$ 25$ WPflG).

Die Wehrpflicht galt, wie in den meisten Ländern zu dieser Zeit, nur für den männlichen Teil der Bevölkerung. Dieser wurde unter der Androhung von Zwangsmassnahmen zum Wehrdienst oder Ersatzdienst gesetzlich verpflichtend. Lange Zeit wurde anhand einer mündlichen Befragung geprüft, ob der Wunsch nach Ersatzdienst aus Gewissensgründen gerechtfertigt ist, was im negativen Fall dazu führen konnte, dass Wehrdienst abzuleisten war, weil die Gewissengründe als nicht stichhaltig angesehen wurden. Wer weder Ersatzdienst noch Wehrdienst leisten wollte, galt laut $\$ 16 \mathrm{WPflG}$ als fahnenflüchtig und konnte mit einer Freiheitsstrafe bis zu fünf Jahren bestraft werden. Als Begründung für die Wehrpflicht in Deutschland galt zum einen, den personellen Bedarf der Bundeswehr decken zu können, zum anderen sollte aufgrund der deutschen Geschichte ein enges Band zwischen der Bevölkerung und den Streitkräften existieren. Der Begriff, der sich in diesem Zusammenhang einbürgerte, war der Bürger in Uniform. Unter allen Umständen sollte ein Eigenleben der Armee als Staat im Staate vermieden und die Bundeswehr fest im demokratischen Staat verankert werden (Werkner 2011).

Die Begründung für die Wehrpflicht basiert auf der zu Beginn beschriebenen Haltung den Schutz des liberal-demokratischen Staates als höheres Gut und übergeordnete Ziel zu betrachten. Dafür darf im schlimmsten Fall auch die Freiheit des Individuums innerhalb dieses Staates beschränkt werden.

In Deutschland wurde die Wehrpflicht am 1. Juli 2011 ausgesetzt (Deutscher Bundestag 2011). Mit der Aussetzung sollte signalisiert werden, dass die Wehrpflicht an sich immer noch ihre Berechtigung hat, aber mit der Umstrukturierung der Streitkräfte und sicherheitspolitisch sei diese nicht mehr begründbar.

\section{Wehrpflicht in den USA}

Die Vereinigten Staaten von Amerika haben sich in Ihrer Geschichte zumeist auf eine Freiwilligenarmee gestützt. Ausnahmen waren grosse Kriege und die Zeit zwischen 1948 und 1973, wie die President's Commission on an All-Volunteer Armed Force bemerkt (CoAVA 1970). Die Begründung für die Existenz der Armee selbst, aber auch für die Wehrpflicht, ist die Verteidigung von Freiheit und Gerechtigkeit:

"Since the founding of the republic, a primary task of the government of the United States has been to provide for the common defense of a society established to secure the blessings of liberty and justice" (CoAVA 1970: 5).

Zur Verteidigung dieser Freiheit schien es gerechtfertigt, in besonderen Zeiten die Freiheit der Bürger zu beschneiden und sie zum Dienst an der Waffe zu zwingen. Wer sich der Einberufung durch die Armee entzog, konnte mit einer Freiheitsstrafe von bis zu 5 Jahren und/oder einer Geldstrafe von bis zu US\$10’000 belegt werden (Worthington 2015).

Interessanterweise thematisiert die CoAVA das Dilemma zwischen der Verteidigung der Freiheit des Landes und der Beschränkung der Freiheit seiner Bürger explizit: 
"A return to an all-volunteer force will strengthen our freedoms, remove an inequity now imposed on the expression of the patriotism that has never been lacking among our youth, promote the efficiency of the armed forces, and enhance their dignity. It is the system for maintaining standing forces that minimizes government interference with the freedom of the individual to determine his own life in accord with his values" (CoAVA 1970: 5).

Zwar habe jeder Bürger die moralische Verpflichtung, sein Land zu verteidigen, aber es sei auch offensichtlich, dass die individuelle Freiheit beschnitten würde. Trotz verschiedener Bedenken bezüglich der Auswirkungen einer Abschaffung der Wehrpflicht in Bezug auf die Zusammensetzung und Steuerbarkeit der Armee sowie der Befürchtung, eine Freiwilligenarmee würde den Gedanken der Verpflichtung zur Verteidigung des Landes unterminieren (CoAVA 1970: 5), wurde die Wehrpflicht im Jahre 1973 abgeschafft. Im Jahr 1974 implementierte Präsident Ford eine Amnestie für die damaligen Verweigerer des Wehrdienstes, unter der Bedingung der Durchführung eines alternativen Dienstes zwischen 6 und 24 Monaten (Associated Press 1974: 9). Bis heute sind Männer zwischen 18 und 26 Jahren aber verpflichtet, sich beim Selective Service System registrieren zu lassen. Wer dem nicht nachkommt, kann mit bis zu 5 Jahren Freiheitsstrafe und/oder einer Geldstrafe von bis zu US\$10'000 bestraft werden. Allerdings wurde seit 1986 niemand mehr für dieses Vergehen verfolgt oder bestraft (Resistance News o.J.).

\section{Wehrpflicht in der Schweiz}

Die Wehrpflicht in der Schweiz, Militärdienstpflicht genannt, besteht seit 1874 und ist eine in der Bundesverfassung verankerte Pflicht der männlichen Bürger der Schweiz. Artikel 59 Absatz 1 der Bundesverfassung sieht vor: „Jeder Schweizer ist verpflichtet, Militärdienst zu leisten. Das Gesetz sieht einen zivilen Ersatzdienst vor." (Art. 59 Abs. 1 BV).

Der Bundesrat vertrat in seiner Botschaft zur Volksinitiative Ja zur Abschaffung der Wehrpflicht im Jahr 2012 die Auffassung, dass der Militärdienst eine Pflicht des Bürgers sei:

„Grundidee der Militärdienstpflicht ist es, dass der Staat zur Abwehr von existenziellen Bedrohungen auf die persönliche Leistung seiner Bürger zurückgreifen kann. Mit Blick auf Extremsituationen darf der Staat also zu einer Zwangsmassnahme greifen. Dieser Zwang ist legitimiert durch das Ziel, die Bevölkerung vor existenziellen Bedrohungen zu schützen" (Bundesrat 2012: 8314).

Das Dilemma zwischen Verteidigung des Staates und der Freiheit der Bürger und Bürgerinnen wird auch hier thematisiert und eindeutig im Sinne der Verteidigung des Staates formuliert. Da dieser Zwang auch demokratisch kontrolliert sei, würde auch die notwendige Kontrolle erfolgen (Bundesrat 2012: 8314).

Schliesslich verweist der Bundesrat noch auf Artikel 4 der Konvention vom 4. November 1950 zum Schutz der Menschenrechte und Grundfreiheiten sowie das Völkerrecht, um die Legitimität dieses Zwangs zu unterstreichen:

„Artikel 4 der Konvention vom 4. November 1950 zum Schutz der Menschenrechte und Grundfreiheiten (Europäische Menschenrechtskonvention, EMRK) anerkennt diese Form staatlichen Zwangs ausdrücklich. Die Militärdienstpflicht ist völkerrechtlich zulässig. Sie ist 
mit einer freiheitlichen Ordnung vereinbar, demokratisch legitimiert und in der Schweiz staatspolitisch fest verankert. Der Bundesrat vermag in der Militärdienstpflicht keinen überholten Zwang zu erkennen" (Bundesrat 2012: 8314).

Schliesslich hebt der Bundesrat noch den gesellschaftlichen Nutzen des Wehrdienstes hervor, da dieser eine Begegnungsplattform unterschiedlichster Bevölkerungsgruppen sei:

„Militärdienstpflicht und Miliz schaffen ein demokratisches Wehrmodell, das zum persönlichen Engagement verpflichtet, unabhängig vom sozialen und wirtschaftlichen Hintergrund.

(...) In der Armee begegnen sich Bürger mit unterschiedlichen politischen Auffassungen aus allen Landesteilen und sozialen Schichten" (Bundesrat 2012: 8314).

Das individuelle Freiheitsrecht der Bürgerinnen und Bürger wird auch hier zugunsten der Verteidigung des Staates und des Zusammenhaltes der Gesellschaft hintangestellt, nicht aber ohne den Zwangscharakter des Militärdienstes zu problematisieren.

Darüber hinaus war, ähnlich wie in den USA, bis zum Jahr 1996 die Möglichkeit eines Ersatzdienstes nicht gegeben. Bürger, die aus ethischen oder auch anderen Gründen keinen Dienst an der Waffe leisten wollten oder konnten, wurden dementsprechend einer Strafverfolgung unterzogen. Ab 1992 wurde durch die so genannte Barras-Reform (Historisches Lexikon der Schweiz o.J.) eine Möglichkeit innerhalb des Militärs geschaffen, alternativ eine Arbeitsleistung zu erbringen, um den Militärdienst umgehen zu können. Die Möglichkeit Zivildienst zu leisten, wurde dagegen erst durch eine Volksinitiative 1992 geschaffen. Die Umsetzung erfolgte im Zivildienstgesetz 1995 (Bundesrat 2012).

\section{Folgen für die Verweigerer in der Schweiz}

Die fehlende Möglichkeit, dem Dienst an der Waffe aus dem Weg zu gehen, hatte teils dramatische Folgen für die Betroffenen. Seit den sechziger Jahren stieg die Zahl der Verurteilungen kontinuierlich an. Waren es 1961 noch 47 Verurteilungen, so sind 1970 schon 175 und 1975 sogar 520 Verurteilungen wegen Dienstverweigerung verzeichnet (Koller 2008), im Jahre 1984 erreichte die Anzahl der Verweigerungen mit 788 ihre Höchstzahl (Historisches Lexikon der Schweiz o.J.). Insgesamt gab es zwischen 1947 und 1996 über 13 000 Fälle von Militärdienstverweigerungen (Burri 2005; Möcklin 1998), viele der Betroffenen wurden zu Freiheitsstrafen verurteilt und mussten diese auch, zumindest teilweise, absitzen. Die Folgen für die von den Strafen betroffenen Personen waren dramatisch: Sie galten als vorbestraft, sahen sich Restriktion ausgesetzt, wenn sie für den Staat arbeiten wollten und kämpften lange Jahre mit den psychischen Folgen der Inhaftierung, insbesondere mit Depressionen (Frenkel 2017). In kleineren Gemeinden wurden Dienstverweigerer oftmals geschnitten, zudem wurden Dossiers über sie angelegt (Frenkel 2017). Die eigentliche Bestrafung ging also mit einer gesellschaftlichen Diskriminierung einher. Bekannt sind Fälle von Verweigerung einer Anstellung im Schuldienst, Diskriminierung durch den Schweizer Alpen-Club wegen angeblicher mangelnder Ehrenhaftigkeit, eine Kündigung bei der SBB wegen mangelnder Leistungsbereitschaft oder auch die Verweigerung von Krediten (Frenkel 2017).

Der Fall Schweiz macht besonders deutlich, dass Gesellschaften zur Verteidigung ihrer Freiheit oft bereit waren und sind, die persönlichen Rechte ihrer Bürgerinnen und Bürgerinnen zu beschneiden, sie zu bestrafen und auch gesellschaftlich zu negieren. Dies ist im schwei- 
zerischen Fall besonders problematisch, da bis ins Jahr 1992 überhaupt keine Möglichkeit bestand, dem Dienst an der Waffe zu entgehen, ein tatsächlicher Zivildienst wurde sogar erst 1996 implementiert. Während die Eidgenossenschaft aber bis zum heutigen Tage an der grundsätzlichen Pflicht zum Dienst an der Waffe für die Gesellschaft festhält, hat sie schon lange ihre Haltung bezüglich des Zivildienstes geändert. Schliesslich heisst es in Artikel 1 des Zivildienstgesetzes: „Militärdienstpflichtige, die den Militärdienst mit ihrem Gewissen nicht vereinbaren können, leisten auf Gesuch hin einen länger dauernden zivilen Ersatzdienst (Zivildienst) nach diesem Gesetz" (Art.1 ZDG).

Die Eidgenossenschaft erkennt mit dem Zivildienstgesetz an, dass ein moralisches Recht existiert, den Dienst an der Waffe zu verweigern. Allen Verweigerern wurde dieses Recht und diese Anerkennung bis 1992 verweigert, mehr noch, sie wurden bestraft und diskriminiert. Bis heute ist keine Entschuldigung oder gar Entschädigung erfolgt. Man fragt sich, warum.

\section{Literaturverzeichnis}

Associated Press (1974). Flexible Amnesty Plan is Reported Set by Ford. New York Times, 14.09.1974, 9.

Bundesrat (2012). Botschaft zur Volksinitiative «Ja zur Aufhebung der Wehrpflicht. Bern: Bundesrat.

Bundesverfassung der Schweizerischen Eidgenossenschaft (BV), vom 18.April 1899 (Stand am 1.Januar 2016). Internet-Seite. Zugriff am 30.03 .17 auf https://www.admin.ch/opc/de/classified-compilation/19995395/index.html.

Burri, Samuel (2005). "Im Willen zum Frieden sind wir uns einig.» - Eine Untersuchung der Prozesse gegen Militärdienstverweigerer in der Deutschschweiz zu Beginn der 1970er Jahre. Lizentiatsarbeit. Historisches Institut, Universität Bern, Bern.

CoAVA (1970). The Report of the President's Commission on an All-Volunteer Armed Force. London: Collier Books/Macmillan.

Deutscher Bundestag (2011). Aussetzung der allgemeinen Wehrpflicht beschlossen. Internet-Seite. Zugriff am 15.01.17 auf https://www.bundestag.de/dokumente/textarchiv/2011/33831649_kw12_de_wehrdienst.

Europäische Menschenrechtskonvention (EMRK). Konvention vom 4. November 1950 zum Schutz der Menschenrechte und Grundfreiheiten. Internet-Seite. Zugriff am 15.01.17 auf https://www.menschenrechtskonvention.eu/konvention-zum-schutz-der-menschenrechte-undgrundfreiheiten-9236/.

Frenkel, Beni (2017). Der Krieg der Pazifisten. Tages-Anzeiger, 06.01.2017.

Historisches Lexikon der Schweiz (o.J.). Dienstverweigerung. Internet-Seite. Zugriff am 15.01.17 auf http://www.hls-dhs-dss.ch/textes/d/D8678.php. 
Koller, Christian (2008). Der lange Weg zum „zivilen Ersatzdienst“ in der Schweiz. In: Müller, Christian Th. und Dierk Walter (Hrsg.) (2008): Ich dien'nicht! Wehrdienstverweigerung in der Geschichte (227-242). Berlin: dissertation.de-Verlag.

Möcklin, Emanuel (1998). Militärdienstverweigerung. Über die Ursachen der Militärdienstverweigerung in der Schweiz. Eine explorative Studie. Lizentiatsarbeit. Soziologisches Institut, Universität Zürich.

Narr, Wolf-Dieter (2008). Kapitel 3. Der CDU-Staat (1949-66). In: Roth, Roland und Dieter Rucht (Hrsg.) (2008). Die Sozialen Bewegungen in Deutschland seit 1945: Ein Handbuch (347-362). Frankfurt a.M.: Campus.

Deutscher Bundestag (2011). Aussetzung der allgemeinen Wehrpflicht beschlossen. Internet-Seite. Zugriff am 15.01.17 auf https://www.bundestag.de/dokumente/textarchiv/2011/33831649_kw12_de_wehrdienst.

Resistance News (o.J.). Prosecutions of Draft Registration Resisters. Internet-Seite. Zugriff am 15.01.17 auf https://hasbrouck.org/draft/prosecutions.html.

Wehrpflichtgesetz (WPflG) vom 21.Juli 1956. Bundesgesetzblatt, 36, 651-661.

Werkner, Ines-Jacqueline (2011). Wehrpflicht und Zivildienst - Bestandteile der politischen Kultur? Aus Politik und Zeitgeschichte, 48, 39-45.

Worthington, Daryl (2015). Carter Pardons Vietnam Dodgers. New Historian. Internet-Seite. Zugriff am 15.01.17 auf http://www.newhistorian.com/carter-pardons-vietnam-draft-dodgers/2758/

Zivildienstgesetz (ZDG) vom 6. Oktober 1995 (Stand am 1. Juli 2016). Internet-Seite. Zugriff am 15.01.17 auf https://www.admin.ch/opc/de/classified-compilation/19950281/. 